SHU-Pre2009-08

arXiv:0905.2675[hep-th]

August, 2009

\title{
Shear viscosity and instability from third order Lovelock gravity
}

\author{
Xian-Hui GE* SANG-Jin Sin ${ }^{\dagger}$, ShaO-Feng Wu* \\ and GuO-Hong YANG* \\ *Department of Physics, Shanghai University, \\ Shanghai 200444, China \\ gexh@shu.edu.cn, sfwu@shu.edu.cn,ghyang@shu.edu.cn \\ † Department of Physics, Hanyang University, Seoul 133-791, Korea \\ sjsin@hanyang.ac.kr
}

\begin{abstract}
We calculate the ratio of shear viscosity to entropy density for charged black branes in third order Lovelock theory. For chargeless black branes, the result turns out to be consistent with the prediction made in arXiv:0808.3498[hep - th]. We find that, the third order Lovelock gravity term does not contribute to causality violation unlike the Gauss-Bonnet term. The stability of the black brane again requires the value of the Lovelock coupling constant to be bounded by $1 / 4$ in the infinite dimensionality limit.
\end{abstract}

\section{Introduction}

The AdS/CFT correspondence [1-3] provides an interesting theoretical framework for studying relativistic hydrodynamics of strongly coupled gauge theories. The result of RHIC experiment on the viscosity/entropy ratio turns out to be in favor of the prediction of AdS/CFT [4-6]. Some attempt has been made to map the entire process of RHIC experiment in terms of gravity dual [7]. The way to include chemical potential in the theory was figured out in $[8,9]$. 
It had been conjectured that the viscosity value of theories with gravity dual may give a lower bound for the $\eta / s=\frac{1}{4 \pi}$ for all possible liquid [14]. However, in the presence of higher-derivative gravity corrections, the viscosity bound and causality are also violated as a consequence [15-18]. The ratio of shear viscosity to entropy density are of particular interest in higher derivative gravity duals because those higher derivative terms can be regarded as generated from stringy corrections given the vastness of the string landscape. In [19-21], the authors computed $\eta / s$ for general gravity duals by determining the ratio of two effective gravitational couplings. The $\eta / s$ in presence of arbitrary $R^{2}$ and $R^{3}$ terms in the bulk action were calculated in [22].

The higher derivative terms may be a source of inconsistencies because higher powers of curvature could give rise to fourth or even sixth order differential equation for the metric, and in general would introduce ghosts and violate unitarity. Zwiebach and Zumino $[23,24]$ found that ghosts can be avoided if the higher derivative terms only consist of the dimensional continuations of the Euler densities, leading to second order field equations for the metric. These theories are the so called Lovelock gravity [25]. The zeroth order of Lovelock gravity correspondences to the cosmological constant. The first order is the Einstein equation and the second order correspondences to Gauss-Bonnet theory. Higher derivative effects on $\eta / s$ in the presence of a chemical potential have been discussed in [26-33]. In this paper, we discuss shear viscosity in third order Lovelock gravity.

Our motivation for this paper is based on the following facts:

1). Although people expect that $\eta / s$ might receive corrections from third and higher order Lovelock terms, it was conjectured in [19] that $\eta / s$ gets no corrections at all for higher order Lovelock terms except the Gauss-Bonnet terms. In this paper, we compute $\eta / s$ for third order Lovelock gravity directly by using the standard method developed in $[4,5]$ and compare our result with that of [19].

2). In [15] and [16], the authors showed that if we consider the Gauss-Bonnet correction to Einstein equation, the viscosity bound is violated in the hydrodynamics regime. Moreover, causality violation happens in the high frequency regime $\left(k^{\mu} \rightarrow \infty\right)$, which implies that theories in that regime are pathological [17]*. In [26] and [27], some of us considered medium effect and the higher derivative correction simultaneously by adding charge and Gauss-Bonnet terms and found that the viscosity bound as well as causality violation is

\footnotetext{
${ }^{*}$ The causality issue in Gauss-Bonnet gravity was further studied in [34].
} 
not changed by the charge. After adding the third order Lovelock terms, the causality structure would be different from that of the Gauss-Bonnet gravity.

3). It is worth to studying the stability of black branes (black holes) in third order Lovelock gravity in that the stability can constrain the higher derivative coupling constants. The instability of $D$-dimensional asymptotically flat Einstein-Gauss-Bonnet and Lovelock black holes has been discussed by several authors [35-38]. Their results show that for gravitational perturbations of Schwarzschild black holes in $D \geq 5$ Gauss-Bonnet gravity, instability occurs only for $D=5$ and $D=6$ cases at large value of $\alpha^{\prime}$ [36]. In [37], the authors showed that small black holes in Lovelock gravity are unstable. In this paper, we extend our previous computation to third order Lovelock gravity in $D$-dimensional spacetime and show how stability constrains the Lovelock coupling constant.

The plan of this paper is as follows. In section 2, we briefly review the thermodynamic properties of Reissner-Nordström-AdS black brane solution in third order Lovelock gravity. In section 3, we compute the viscosity to entropy density ratio via Kubo formula and its charge dependence. In section 4, the causality problem is discussed. We study the stability issue of Reissner-Nordström-AdS black branes in third order Lovelock gravity in section 5 . Conclusions and discussions are presented in the last section.

\section{Reissner-Nordström-AdS black brane in third order Lovelock gravity}

We start by introducing the following action in $D$ dimensions which includes Lovelock terms and $U(1)$ gauge field:

$$
I=\frac{1}{16 \pi G_{D}} \int \mathrm{d}^{D} x \sqrt{-g}\left(-2 \Lambda+\mathcal{L}_{1}+\alpha_{2}^{\prime} \mathcal{L}_{2}+\alpha_{3}^{\prime} \mathcal{L}_{3}-4 \pi G_{D} F_{\mu \nu} F^{\mu \nu}\right),
$$

where

$$
\begin{aligned}
& \mathcal{L}_{1}=R \\
& \mathcal{L}_{2}=R_{\mu \nu \gamma \delta} R^{\mu \nu \gamma \delta}-4 R_{\mu \nu} R^{\mu \nu}+R^{2}, \\
& \mathcal{L}_{3}=2 R^{\mu \nu \sigma \kappa} R_{\sigma \kappa \rho \tau} R_{\mu \nu}^{\rho \tau}+8 R_{\sigma \rho}^{\mu \nu} R_{\nu \tau}^{\sigma \kappa} R_{\mu \kappa}^{\rho \tau}+24 R^{\mu \nu \sigma \kappa} R_{\sigma \kappa \nu \rho} R_{\mu}^{\rho}+3 R R^{\mu \nu \sigma \kappa} R_{\sigma \kappa \mu \nu} \\
& +24 R^{\mu \nu \sigma \kappa} R_{\sigma \mu} R_{\kappa \nu}+16 R^{\mu \nu} R_{\nu \sigma} R_{\mu}^{\sigma}-12 R R^{\mu \nu} R_{\mu \nu}+R^{3},
\end{aligned}
$$

$\Lambda$ is the cosmological constant, $\alpha_{2}^{\prime}$ and $\alpha_{3}^{\prime}$ are Gauss-Bonnet and third order Lovelock coefficients, respectively. The field strength is defined as $F_{\mu \nu}(x)=\partial_{\mu} A_{\nu}(x)-\partial_{\nu} A_{\mu}(x)$. 
The thermodynamics and geometric properties of black objects in Lovelock gravity were studied in several papers [39-42]. From the action (2.1), we can write down the equation of motion [43],

$$
\sum_{k=0}^{3} \frac{1}{2^{k+1}} c_{k} \delta_{\nu e_{1} \ldots e_{k} f_{1} \ldots f_{k}}^{\mu c_{1} \ldots c_{k} d_{1} \ldots d_{k}} R_{c_{1} d_{1}}^{e_{1} f_{1}} \cdots R_{c_{k} d_{k}}^{e_{k} f_{k}}=8 \pi G_{D} T_{\nu}^{\mu}
$$

where $T_{\nu}^{\mu}=F_{\rho}^{\mu} F_{\nu \sigma} g^{\rho \sigma}-\frac{1}{4} g^{\mu}{ }_{\nu} F_{\rho \sigma} F^{\rho \sigma}$. Note that for third order Lovelock gravity, we must deal with $D$-dimensional spacetimes with $D \geq 7$.

If we choose

$$
\alpha_{2}^{\prime}=\frac{\alpha}{(D-3)(D-4)}, \quad \alpha_{3}^{\prime}=\frac{\alpha^{2}}{3(D-3) \cdots(D-6)},
$$

the charged black hole solution in $D$ dimensions for this action is described by [38]

$$
\begin{aligned}
\mathrm{d} s^{2} & =-H(r) N^{2} \mathrm{~d} t^{2}+H^{-1}(r) \mathrm{d} r^{2}+\frac{r^{2}}{l^{2}} h_{i j} \mathrm{~d} x^{i} \mathrm{~d} x^{j} \\
A_{t} & =-\frac{Q}{4 \pi(D-3) r^{D-3}}
\end{aligned}
$$

with

$$
H(r)=k_{0}+\frac{r^{2}}{\alpha}\left\{1-\left[1-\frac{3 \alpha}{l^{2}}\left(1-\frac{m l^{2}}{r^{D-1}}+\frac{q^{2} l^{2}}{r^{2 D-4}}\right)\right]^{1 / 3}\right\}, \quad \Lambda=-\frac{(D-1)(D-2)}{2 l^{2}},
$$

where the parameter $l$ corresponds to AdS radius. The constant $N^{2}$ will be fixed later. Note that the constant value of $k_{0}$ can be \pm 1 or 0 and $h_{i j} \mathrm{~d} x^{i} \mathrm{~d} x^{j}$ represents the line element of a $(D-2)$-dimensional hypersurface with constant curvature $(D-2)(D-3) k_{0}$ and volume $V_{D-2}$. The gravitational mass $M$ and the charge $Q$ are expressed as

$$
\begin{aligned}
M & =\frac{(D-2) V_{D-2}}{16 \pi G_{D}} m, \\
Q^{2} & =\frac{2 \pi(D-2)(D-3)}{G_{D}} q^{2} .
\end{aligned}
$$

Taken the limit $\alpha_{2}^{\prime}, \alpha_{3}^{\prime} \rightarrow 0$ with $k_{0}=0$, the solution corresponds to one for ReissnerNordström-AdS (RN-AdS). The hydrodynamic analysis in this background has been done in $[44,45]$.

One may notice that here we use a black hole solution by choosing particular values of $\alpha_{2}^{\prime}$ and $\alpha_{3}^{\prime}$ so that our computation can be simplified greatly. Eq.(2.3) with the choice 
(2.4) yields one real and two complex solutions. We use the real solution in (2.5a). The general solution of third order Lovelock gravity in $D$ dimensions for any arbitrary values of $\alpha_{2}^{\prime}$ and $\alpha_{3}^{\prime}$ was obtained in [38], but the line element of the metric turns out to be very complicated. Furthermore, the general solution may present naked singularities, which is not what we are interested [38]. In this paper, we only focus on the special case given in (2.5a).

In the following, we mainly focus on $D$-dimensional case with $k_{0}=0$. Defining $\lambda=\alpha / l^{2}$, the function $H(r)$ becomes

$$
H(r)=\frac{r^{2}}{\lambda l^{2}}\left\{1-\left[1-3 \lambda\left(1-\frac{r_{+}^{D-1}}{r^{D-1}}-a \frac{r_{+}^{D-1}}{r^{D-1}}+a \frac{r_{+}^{2 D-4}}{r^{2 D-4}}\right)\right]^{1 / 3}\right\}
$$

where $a=\frac{q^{2} l^{2}}{r_{+}^{2 D-4}}$. The event horizon is located at $r=r_{+}$. The constant $N^{2}$ in the metric (2.5a) can be fixed at the boundary whose geometry would reduce to flat Minkowski metric conformaly, i.e. $\mathrm{d} s^{2} \propto-c^{2} \mathrm{~d} t^{2}+\mathrm{d} \vec{x}^{2}$. On the boundary $r \rightarrow \infty$, we have

$$
H(r) N^{2} \rightarrow \frac{r^{2}}{l^{2}}
$$

so that $N^{2}$ is found to be

$$
N^{2}=\frac{\lambda}{1-(1-3 \lambda)^{1 / 3}} .
$$

Note that the boundary speed of light is specified to be unity $c=1$. From (2.6), one can assume $\lambda \leq 1 / 3$ in order to work with the branch of well-behaved solutions. That is because when $\lambda>1 / 3$ the sign of $H(r)$ becomes minus in the asymptotic infinity, and we cannot recover the AdS geometry**. In section 4, we will carry out the causality analysis and find the causality constraints imposed on the value of $\lambda$.

We shall give thermodynamic quantities of this background. The temperature at the event horizon is defined as

$$
T=\frac{1}{2 \pi \sqrt{g_{r r}}} \frac{\mathrm{d} \sqrt{g_{t t}}}{\mathrm{~d} r}=\frac{N r_{+}}{4 \pi l^{2}}[(D-1)-(D-3) a] .
$$

*In the Gauss-Bonnet case, the function $H(r)$ has a different from: $H(r) \quad=$ $\frac{r^{2}}{2 \lambda l^{2}}\left\{1-\sqrt{1-4 \lambda_{G B}\left(1-\frac{r_{+}^{2}}{r^{2}}\right)\left(1-\frac{r_{-}^{2}}{r^{2}}\right)\left(1-\frac{r_{0}^{2}}{r^{2}}\right)}\right\}$, which implies that the significant value of $\lambda$ lies in the range $\lambda_{G B} \leq 1 / 4$. Beyond this point, the Einstein-Maxwell-Gauss-Bonnet action does not admit a vacuum AdS solution, and then the AdS/CFT correspondence is undefined. In [27], it was found that causality requires exactly $\lambda_{G B} \leq 1 / 4$ in the $D \rightarrow \infty$ limit. This result matches precisely the assumption (i.e. $\left.\lambda_{G B} \leq 1 / 4\right)$ used in $[16,32]$. 
The black brane approaches extremal when $a \rightarrow \frac{D-1}{D-3}$ (i.e. $T \rightarrow 0$ ). The entropy of black branes with $k_{0}=0$ obeys the area law [38] and thus the entropy density has the form,

$$
s=\frac{r_{+}^{D-2}}{4 G_{D} l^{D-2}} .
$$

\section{Viscosity to entropy density ratio}

We explored the charge dependence of $\eta / s$ in the presence of Gauss-Bonnet terms for Ddimensional AdS black branes in [27]. In this section, we generalize the previous result on $\eta / s[26,27]$ to third order Lovelock gravity. It is convenient to introduce coordinate in the following computation

$$
\begin{aligned}
& z=\frac{r}{r_{+}}, \quad \omega=\frac{l^{2}}{r_{+}} \bar{\omega}, \quad k_{3}=\frac{l^{2}}{r_{+}^{2}} \bar{k}_{3}, \quad f(z)=\frac{l^{2}}{r_{+}^{2}} H(r), \\
& f(z)=\frac{z^{2}}{2 \lambda}\left[1-\left(1-3 \lambda\left(1-\frac{a+1}{z^{D-1}}+\frac{a}{z^{2 D-4}}\right)\right)^{1 / 3}\right]
\end{aligned}
$$

We now study the tensor type perturbation $h_{x_{2}}^{x_{1}}\left(t, x_{3}, z\right)=\phi\left(t, x_{3}, z\right)$ on the black brane background of the form

$$
d s^{2}=-f(z) N^{2} \mathrm{~d} t^{2}+\frac{\mathrm{d} z^{2}}{f(z)}+\frac{z^{2}}{l^{2}}\left(2 \phi\left(t, x_{3}, z\right) \mathrm{d} x_{1} \mathrm{~d} x_{2}+\sum_{i=1}^{D-2} \mathrm{~d} x_{i}^{2}\right)
$$

Using Fourier decomposition

$$
\phi\left(t, x_{3}, z\right)=\int \frac{\mathrm{d}^{D-1} k}{(2 \pi)^{D-1}} \mathrm{e}^{-i \bar{\omega} t+i \bar{k}_{3} x_{3}} \phi(k, z),
$$

and expanding the action for tenor type gravitational perturbations $\phi\left(t, x_{3}, z\right)$ to the second order, we obtain the effective action in the momentum space

$$
S=\frac{1}{16 \pi G_{D}} \int \frac{d \omega d k_{3}}{(2 \pi)^{D-3}} d z \sqrt{-g}\left(M(z) \phi^{\prime} \phi^{\prime}+M_{2}(z) \phi^{2}\right)
$$

where the prime denotes the derivative with respect to $z$.

An easy way to obtain the equation of motion of the tensor type perturbation is to substitute the fluctuated metric into Eq. (2.3). One then find the linearized equation of motion for $\phi(z)$ from the third order Lovelock field equation:

$$
M(z) \phi^{\prime \prime}(z)+M^{\prime}(z) \phi^{\prime}(z)+M_{2} \phi(z)=0
$$


where

$$
\begin{aligned}
& M(z)=z^{D-2} f\left\{1-\frac{2 \lambda}{D-3}\left[z^{-1} f^{\prime}+z^{-2}(D-5) f\right]+\frac{\lambda^{2} z^{-3}}{D-5}\left[2 f^{\prime}+(D-7) z^{-1} f^{\prime}\right] f\right\} \\
& M_{2}=M(z) \frac{\omega^{2}}{N^{2} f^{2}}-k_{3}^{2} z^{D-4} \times \\
& \left\{1-\frac{2 \lambda}{(D-3)(D-4)}\left(f^{\prime \prime}+(D-5)(D-6) z^{-2} f+2(D-5) z^{-1} f^{\prime}\right)\right. \\
& \left.+\frac{2 \lambda^{2}}{(D-3)(D-4)}\left[z^{-3}\left(f^{\prime 2}+f f^{\prime \prime}\right)+2(D-7) z^{-4} f f^{\prime}+\frac{1}{2}(D-7)(D-8) z^{-5} f^{2}\right]\right\},(3 .
\end{aligned}
$$

We would like to emphasize that when $D=5$ and the $\lambda^{2}$ terms vanished, (3.3) reduces to the main equation obtained in $[16,26]$. The shear viscosity involves physics in the lower frequency and lower momentum limit and one can neglect the $M_{2}(z)$ term in solving Eq.(3.3). For the convenient calculation of the shear viscosity, we would like to introduce a new variable $u=\frac{1}{z}$ and rewrite equation $(3.3)$ in the new coordinate

$$
J(u) \phi^{\prime \prime}(u)+J^{\prime}(u) \phi^{\prime}(u)+J_{2}(u) \phi(u)=0,
$$

where $J(u)=\frac{M(1 / z)}{z^{2}}$ and $J_{2}(u)=M_{2}\left(\frac{1}{z}\right)$.

In order to solve the equation of motion (3.5) in hydrodynamic regime, let us assume that the solution yields

$$
\phi(u)=(1-u)^{\nu} F(u)
$$

where $F(u)$ is regular at the horizon. $\quad \nu= \pm i \frac{\omega}{4 \pi T}$ can be fixed by substituting (3.6) into the equation of motion, which we choose

$$
\nu=-i \frac{\omega}{4 \pi T}
$$

Since we only need to know the behavior at $\omega \rightarrow 0$ region, it is sufficient to expand the solution in terms of frequencies up to the linear order of $\nu$

$$
F(u)=F_{0}(u)+\nu F_{1}(u)+\mathcal{O}\left(\nu^{2}, k_{3}^{2}\right)
$$

The equation governing $F_{0}(u)$ goes as

$$
\left[J(u) F_{0}^{\prime}(u)\right]^{\prime}=0
$$

and can be solved as

$$
F_{0}^{\prime}(u)=\frac{C_{1}}{J(u)},
$$


where $C_{1}$ is an integration constant and must be zero as $J(u)$ goes zero at the horizon so that $F_{0}(u)$ is regular at the horizon. Therefore, $F_{0}(u)$ is a constant, i.e. $F_{0}(u)=C$. From the equation at $\mathcal{O}(\nu)$,

$$
\left[J(u) F_{1}^{\prime}(u)\right]^{\prime}-\left(\frac{C}{1-u} J(u)\right)^{\prime}=0,
$$

we find that the solution can be written as

$$
F_{1}^{\prime}(u)=\frac{C}{1-u}+\frac{C_{2}}{J(u)} .
$$

Regularity of $F_{1}(u)$ at the horizon requires that

$$
C_{2}=-\left[((D-1)-(D-3) a)\left(1-\frac{2 \lambda}{D-3}((D-1)-(D-3) a)\right)\right] C .
$$

The value of $C$ can fixed by the boundary condition $C=\lim _{u \rightarrow 0} \phi(u)=1$. It is worth to noting that the above calculation is same as the Gauss-Bonnet cases given in [26,27].

Using the equation of motion, we write down the on-shell action

$$
I_{\text {on-shell }}=-\left.\frac{r_{+}^{D-1} N}{16 \pi G_{D} l^{D}} \int \frac{\mathrm{d}^{D-1} k}{(2 \pi)^{D-1}}\left(J(u) \phi(u) \phi^{\prime}(u)+\cdots\right)\right|_{u=0} ^{u=1} .
$$

The shear viscosity can be read off using the Kubo formula

$$
\begin{aligned}
\eta & =\lim _{\omega \rightarrow 0} \frac{\operatorname{ImG}(\omega, 0)}{\omega}=\frac{r_{+}^{D-1} N}{16 \pi G_{D} l^{D}} \lim _{\omega \rightarrow 0} \frac{\left.J(u) \phi(u) \phi^{\prime}(u)\right|_{u=0}}{i \omega} \\
& =\frac{1}{16 \pi G_{D}}\left(\frac{r_{+}^{D-2}}{l^{D-2}}\right)\left(1-\frac{2 \lambda}{D-3}[(D-1)-(D-3) a]\right) .
\end{aligned}
$$

The ratio of the shear viscosity to the entropy density turns out to be

$$
\frac{\eta}{s}=\frac{1}{4 \pi}\left(1-\frac{2 \lambda}{D-3}[(D-1)-(D-3) a]\right) .
$$

We obtain the same result as that of [27], which is also consistent with the prediction made in [19] when $a=0$. In other words, the third order Lovelock coupling constant $\alpha_{3}^{\prime}$ (or $\lambda^{2}$ in our case) does not contribute to the shear viscosity.

\section{Causality}

The shear viscosity above is calculated in the hydrodynamical regime ( i.e. $k^{\mu} \rightarrow 0$ ). In this and next sections, we will turn to the high frequency regime (i.e. $k^{\mu} \rightarrow \infty$ ) and discuss the causality issue. 
Due to higher derivative terms in the gravity action, the equation (3.3) for the propagation of a transverse graviton differs from that of a minimally coupled massless scalar field propagating in the same background geometry. Writing the wave function as

$$
\phi(x, u)=\mathrm{e}^{-i \omega t+i k z+i k_{3} x_{3}},
$$

and taking large momenta limit $k^{\mu} \rightarrow \infty$, one can find that the equation of motion (3.3) reduces to

$$
k^{\mu} k^{\nu} g_{\mu \nu}^{\mathrm{eff}} \simeq 0
$$

where the effective metric is given by

$$
\mathrm{d} s_{\text {eff }}^{2}=g_{\mu \nu}^{\text {eff }} \mathrm{d} x^{\mu} \mathrm{d} x^{\nu}=N^{2} f(z)\left(-\mathrm{d} t^{2}+\frac{1}{c_{g}^{2}} \mathrm{~d} x_{3}^{2}\right)+\frac{1}{f(z)} \mathrm{d} z^{2} .
$$

Note that $c_{g}^{2}$ can be interpreted as the local speed of graviton:

$$
c_{g}^{2}(z)=\frac{N^{2} f}{z^{2}} \frac{h_{1}}{h_{2}}
$$

where

$$
\begin{aligned}
h_{1}= & \left\{1-\frac{2 \lambda}{(D-3)(D-4)}\left(f^{\prime \prime}+(D-5)(D-6) z^{-2} f+2(D-5) z^{-1} f^{\prime}\right)\right. \\
& \left.+\frac{2 \lambda^{2}}{(D-3)(D-4)}\left[z^{-3}\left(f^{\prime 2}+f f^{\prime \prime}\right)+2(D-7) z^{-4} f f^{\prime}+\frac{1}{2}(D-7)(D-8) z^{-5} f^{2}\right]\right\}, \\
h_{2}= & \left\{1-\frac{2 \lambda}{D-3}\left[z^{-1} f^{\prime}+z^{-2}(D-5) f\right]+\frac{\lambda^{2} z^{-3}}{D-5}\left[2 f^{\prime}+(D-7) z^{-1} f^{\prime}\right] f\right\} .
\end{aligned}
$$

The above equations can exactly reduce to Gauss-Bonnet cases found in $[16,17]$, if the $\lambda^{2}$ terms vanished. For $D=10$, we can expand $c_{g}^{2}$ near the boundary $\frac{1}{z}=0$,

$$
c_{g}^{2}-1=-\frac{4\left[1-(1-3 \lambda)^{1 / 3}\right]^{2}}{4(1-3 \lambda)^{2 / 3}+2(1-3 \lambda)^{1 / 3}-1}+\mathcal{O}\left(\frac{1}{z}\right) .
$$

We can see that $c_{g}^{2}-1$ is always negative, which implies that the local speed of graviton is smaller than the local speed of light of the boundary CFT without any charge dependence . One can further check that for $D=7,8,9 \ldots$, the first term in $c_{g}^{2}-1$ is also negative. Therefore, from (4.5) we can see that the causality imposes no constraints on possible values of $\lambda$. Figure 1 demonstrates that the value of $c_{g}^{2}$ lies in the region $0.80 \leq c_{g}^{2} \leq-0.8$ 
as a function of $u$ and $\lambda$. Following the procedure of Ref. [17], one can find that the group velocity of the graviton is given by

$$
v_{g}=\frac{\mathrm{d} \omega}{\mathrm{d} k} \sim c_{g}
$$

So different from the Gauss-Bonnet cases [17,26], there is no causality violation in third order Lovelock theory with the particular choice of $\alpha_{2}^{\prime}$ and $\alpha_{3}^{\prime}$. The difference comes from the fact that $\alpha_{3}^{\prime}$ terms change the causal structure of the boundary CFT. For more than third order Lovelock theory, the causal structure might be further modified by $\alpha_{i}^{\prime}(i>3)$.

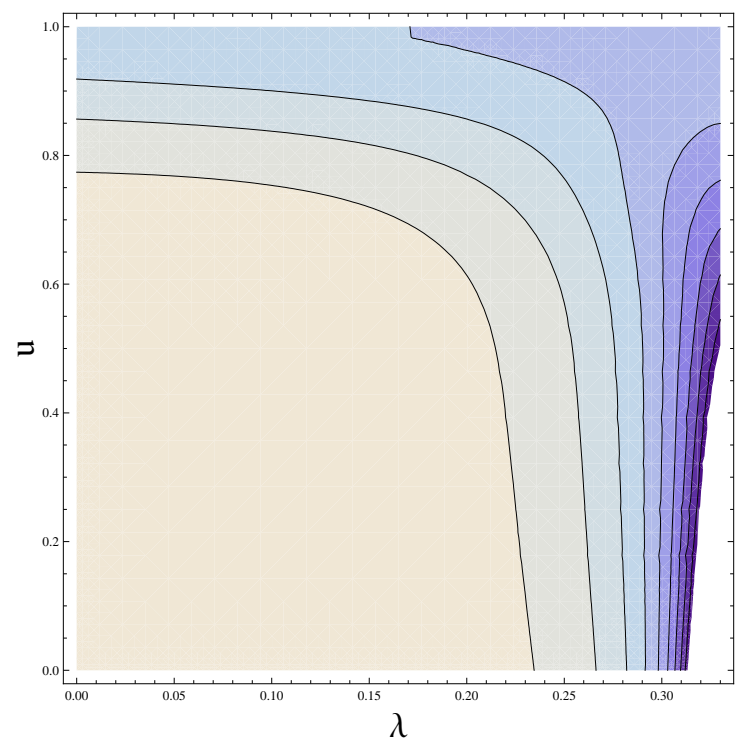

Figure 1: $c_{g}^{2}$ as a function of $u$ and $\lambda$ when we choose $D=7$ and $a=1.4$. The lines correspond to $0.8,0.6, \ldots,-0.8$, respectively, from left to right.

One may notice that our discussions on the causal structure of third order Lovelock gravity are based on the metric (2.5a) and the equation motion in high frequency limit (4.2). Hence, if we consider the general solution of third order Lovelock gravity with arbitrary values of $\alpha_{2}^{\prime}$ and $\alpha_{3}^{\prime}$, we may find totally different causal structure. It remains to be carried out in the future.

\section{Instability}

In section 4, we have demonstrated that for the RN-AdS black brane in third order Lovelock theory, causality violation does not happen, which implies that the results obtained in 
$[17,26,27]$ might not be so universal as we expected. In this section, we extend our previous work on black brane stability to third order Lovelock gravity.

Now, we rewrite the main equation in a Schrödinger form,

$$
-\frac{d^{2} \psi}{d r_{*}^{2}}+V\left(z\left(r_{*}\right)\right) \psi=\omega^{2} \psi, \quad \frac{d r_{*}}{d z}=\frac{1}{N f(z)},
$$

where $\psi\left(z\left(r_{*}\right)\right)$ and the potential is defined by

$$
\begin{aligned}
& \psi=K(z) \phi, \quad K(z) \equiv \sqrt{\frac{M(z)}{N f(z)}}, V=k^{2} c_{g}^{2}+V_{1}(z) \\
& V_{1}(z) \equiv N^{2}\left[\left(f(z) \frac{\partial \ln K(z)}{\partial z}\right)^{2}+f(z) \frac{\partial}{\partial z}\left(f(z) \frac{\partial \ln K(z)}{\partial z}\right)\right]
\end{aligned}
$$

In the large momentum limit, the Schrödinger potential develops a negative gap near the horizon and the negative-valued potential in turn leads to instability of the black brane. In the large momenta limit $k^{\mu} \rightarrow \infty$, the potential is mainly contributed by $k^{2} c_{g}^{2}$. For charged black branes, $c_{g}^{2}$ can be negative near the horizon and the potential is deep enough to have bound states living there. The negative-valued potential yields negative energy eigenvalue (i.e. $\omega^{2}<0$ ). The imaginary part of $\omega$ can then be positive. Substituting the eigenvalue of $\omega$ to the wave function for tensor type perturbations, one immediately find that perturbations grow as time goes on and the black branes thus are unstable. The negative-valued energy bound state corresponds to modes of tachyonic mass Minkowski slices and signals an instability of the black brane [46]. Let us expand $c_{g}^{2}$ in series of $(1-u)$

$$
\begin{aligned}
c_{g}^{2}= & N^{2}[(D-1)-(D-3) a]\left\{D^{2}\left[2 \lambda^{2}(a-1)^{2}+2(a+1) \lambda-1\right]\right. \\
& \left.-D\left[4 \lambda^{2}\left(3 a^{2}-4 a+1\right)+2 \lambda(a+7)-7\right]+\left[\lambda^{2}(3 a-1)^{2}-6 \lambda(a-1)-6\right]\right\} \\
& \{(D-4)[(D-3)-2 \lambda(D-1-(D-3) a)]\}^{-1}(1-u)+\mathcal{O}\left((1-u)^{2}\right) .
\end{aligned}
$$

Since $0 \leq a \leq \frac{D-3}{D-1}$, and $0 \leq u \leq 1, c_{g}^{2}$ will be negative, if

$$
\begin{aligned}
& \left\{D^{2}\left[2 \lambda^{2}(a-1)^{2}+2(a+1) \lambda-1\right]\right. \\
& \left.-D\left[4 \lambda^{2}\left(3 a^{2}-4 a+1\right)+2 \lambda(a+7)-7\right]+\left[\lambda^{2}(3 a-1)^{2}-6 \lambda(a-1)-6\right]\right\} \\
& \{(D-4)[(D-3)-2 \lambda(D-1-(D-3) a)]\}^{-1}<0 .
\end{aligned}
$$


From the above formula, we find the critical value of $\lambda$,

$$
\begin{aligned}
\lambda_{\mathrm{c}} & =\frac{1}{2}\{-(D-1)(D-6)-(D-3)(D+2) a \\
& +\left\{(D-1)^{2}\left(3 D^{2}-26 D+60\right)+(D-3)^{2}\left(3 D^{2}-10 D+28\right) a^{2}\right. \\
& \left.\left.-2 a\left(D^{4}-14 D^{3}+79 D^{2}-174 D+108\right)\right\}^{\frac{1}{2}}\right\}\{D-1-(D-3) a\}^{-2} .
\end{aligned}
$$

Above the line of $\lambda_{c}, c_{g}^{2}$ can be negative. Eq.(5.5) tells us that the stability of the black brane depends on the charge. The minimal value of $\lambda_{c}$ can be obtained in the limit $a \rightarrow\left(\frac{D-1}{D-3}\right)$,

$$
\lambda_{\mathrm{c}, \min }=\frac{1}{4} \frac{(D-3)(D-4)}{(D-1)(D-2)} .
$$

When $D=5$, we recover the result found in [26]. Usually, for the application of AdS/CFT correspondence, we do not need to take infinite dimensionality limit. But the stability of higher dimensional black holes itself is an important topic in the study of black hole physics.

The Einstein-Hilbert action is just the first term in the derivative expansion in a low energy effective theory. The Gauss-Bonnet and the third order Lovelock terms can be regarded as higher order corrections to the Einstein gravity. In this sense, the higher derivative gravity coupling constants should be small. In our discussions, we have found that the coupling constant $\lambda\left(=(D-3)(D-4) \alpha / l^{2}\right)$ depends on the dimensionality $D$. But it seems that for fixed $\alpha$ and AdS radius $l$ as $D$ approaches infinity, $\lambda$ would be very large. That is not what we want. By doing stability analysis, we will find a way to restrict the value of $\lambda$. As the value of $D$ increases, one finds that $\lambda_{c, \text { min }}$ is bounded by $1 / 4$,

$$
\lim _{(D, a) \rightarrow\left(\infty, \frac{D-1}{D-3}\right)} \lambda_{\mathrm{c}}=\frac{1}{4}
$$

Thus we reproduce the result of [27]. Third order Lovelock gravity in our case does not add new constraints on the stability of the black brane. To show explicitly the behavior of gravitational perturbation in higher dimensions $(D \geq 7)$, we solve the Schrödinger equation (5.1) with negative valued potential numerically and find unstable quasinormal modes (QNMs)(see tables 1, 2 and 3).

Table 1 demonstrates that the unstable modes of the black branes are suppressed as $D$ increases. Table 2 and 3 tell us the same story as we found in [27], that is to say, lower value of charge- $(a)$ and $\lambda$ stabilize the perturbation, while the lower value of $D$ strengthens 


\begin{tabular}{|c|c|c|c|c|c|}
\hline$D$ & $\lambda=0.33$ & $\lambda=0.30$ & $\lambda=0.26$ & $\lambda=0.22$ & $\lambda=0.18$ \\
\hline 7 & $35.7830 i$ & $34.2779 i$ & $28.5647 i$ & $21.3057 i$ & $10.0058 i$ \\
8 & $27.8612 i$ & $31.3230 i$ & 22.1646 & $14.6227 i$ & $5.1492 i$ \\
9 & $23.4121 i$ & $22.7276 i$ & $17.9269 i$ & $11.2243 i$ & $3.1228 i$ \\
10 & $22.0265 i$ & $18.5886 i$ & $12.2811 i$ & - & - \\
\hline
\end{tabular}

Table 1: Unstable QNMs for third order charged Lovelock black brane perturbation of tensor type for fixed charge $(a=1.20)$ and $k_{3}=500$. As $D$ increases, the unstable modes are suppressed. And also, small $\lambda$ helps to smooth the perturbation.

\begin{tabular}{|c|c|c|c|c|c|}
\hline$\lambda$ & $a=1.3$ & $a=1.2$ & $a=1.0$ & $a=0.8$ & $a=0.6$ \\
\hline 0.33 & $31.6732 i$ & $27.8612 i$ & $12.7312 i$ & - & - \\
0.30 & $31.5500 i$ & $27.3852 i$ & $9.9506 i$ & - & - \\
0.27 & $28.1982 i$ & $23.7183 i$ & $5.1296 i$ & - & - \\
0.24 & $23.4156 i$ & $18.6473 i$ & - & - & - \\
\hline
\end{tabular}

Table 2: Unstable QNMs for third order charged Lovelock black brane perturbation of tensor type for fixed dimensionality $(D=8)$ and $k_{3}=500$. This table indicates that instability is increased by a chemical potential.

\begin{tabular}{|c|c|c|c|c|}
\hline$D$ & $a=1.4$ & $a=1.3$ & $a=1.2$ & $a=0.8$ \\
\hline 7 & $42.7952 i$ & $39.3407 i$ & $33.3140 i$ & - \\
8 & $30.8934 i$ & $29.5030 i$ & $25.1211 i$ & - \\
9 & - & $23.4155 i$ & $20.6184 i$ & - \\
10 & - & - & $17.442 i$ & - \\
\hline
\end{tabular}

Table 3: Unstable QNMs for third order charged Lovelock black brane perturbation of tensor type for fixed $\lambda(\lambda=0.28)$. This table shows combined effects of $\mathrm{D}$ and the chemical potential. Note that $a=1.4$ exceeds the maximal value of charge permitted for 9- and 10-dimensional charged black brane and thus we leave the frequency blank there. 
the instability. The reason for why higher $D$ suppresses the gravitational fluctuation is because that no matter how big $D$ is, $\lambda$ is bounded by $1 / 4$, which means that for fixed AdS radius $l, \alpha^{\prime} \rightarrow 0$ as the value of $D$ goes up. The upper bound of $\lambda$ constrains the gravitational perturbation in the larger $D$ limit. For QNMs of RN-AdS black holes in Einstein and Gauss-Bonnet gravity, one may refer to [36, 47].

It would be very interesting to check for fixed value of charge, for which value of $\lambda$ the black brane becomes stable. In order to do this, one should first fix $D$ in (5.5), then obtain a formula between $\lambda$ and $a$. Actually, (5.5) indicates that for $\lambda<\lambda_{c}(D, a)$, the black brane becomes stable. For 5-dimensional black brane with charge in Gauss-Bonnet gravity, constraints from causality as well as stability separate the physics into four regions in $(a, \lambda)$ space: consistent region; only causality violation region; only unstable modes region; both causality violation and unstable modes region (see figure 4 in [26] for details). But for the particular case we are considering here, since causality violation does not occur, we have only two phases in the $(a, \lambda)$ space: stable and unstable modes regions marked by (5.5). One thing one need to be aware of is that instability of the black brane does not correspond to any fundamental pathology with the theory. This is quiet different from the causality violation which means that a theory is pathological. In the dual gravitational description, the unstable QNMs is identified with unstable uniform plasma with respect to certain non-uniform perturbation [34].

\section{Conclusions and discussions}

In conclusion, we derive the main equation for tensor type perturbation in third order Lovelock theory and compute the shear viscosity. The result turns out to be in agreement with the prediction made in [19] when $a=0$, that is to say, the third order Lovelock term does not add new ingredients into the shear viscosity of Gauss-Bonnet theory.

We notice that an interesting point comes from the causality analysis. While in the Gauss-Bonnet theory, causality could be violated in the boundary CFT, we do not find causality violation in third order Lovelock theory. From (4.4), we can see that the local speed of graviton depends on both $\alpha_{2}^{\prime}(\sim \lambda)$ and $\alpha_{3}^{\prime}\left(\sim \lambda^{2}\right)$. Although we are working only

with a special choice of $\alpha_{2}^{\prime}$ and $\alpha_{3}^{\prime}$, Eq. (4.4) implies that causality receives corrections from the $\alpha_{3}^{\prime}$ term. Thus, the causal structure in general third order Lovelock gravity must 
be different from the Gauss-Bonnet gravity. We also expect that higher than fourth order Lovelock theory may impose more constraints on the causal structure of the boundary CFT.

The instability of charged black brane with third order Lovelock theory shows the same properties as that of Gauss-Bonnet corrections. We find that higher $D$ suppresses the unstable modes, but larger value of charge and $\lambda$ strengthen the perturbation. As $D$ approaches infinity, the stability requires $\lambda$ to be bounded by $1 / 4$. This is an important observation in that Eq.(2.6) indicates that $\lambda$ could be as big as $1 / 3$ without any causality violation happens in third order Lovelock gravity. But $\lambda \sim 1 / 3$ violates the assumption $\lambda_{G B} \leq 1 / 4$ used in $[16,32]$. Fortunately, after imposing the stability constraint, we can recover the requirement $\lambda \leq 1 / 4$ and thus third order Lovelock and Gauss-Bonnet gravity are somehow consistent.

\section{Acknowledgments}

This work is supported partly by the Shanghai Leading Academic Discipline Project (project number S30105). The work of SFW is partly supported by NSFC under Grant Nos. 10847102, and the Innovation Foundation of Shanghai University. The work of SJS was supported by KOSEF Grant R01-2007-000-10214-0. This work is also supported by Korea Research Foundation Grant KRF-2007-314-C00052 and SRC Program of the KOSEF through the CQUeST with grant number R11-2005-021.

\section{References}

[1] J. M. Maldacena, Adv. Theor. Math. Phys. 2 (1998) 231, [arXiv:hep-th/9711200].

[2] S. S. Gubser, I.R. Klebanov and A.M. Polyakov, Phys. Lett. B428 (1998) 105, [arXiv:hep-th/9802109].

[3] E. Witten, Adv. Theor. Math. Phys. 2 (1998) 253, [arXiv:hep-th/9802150].

[4] G. Policastro, D. T. Son and A.O. Starinets, Phys. Rev. Lett. 87 (2001) 081601, [arXiv:hep-th/0104066]. 
[5] P. Kovtun, D. T. Son and A.O. Starinets, JHEP 0310 (2003) 064, [arXiv:hep-th/0309213].

[6] A. Buchel and J. T. Liu, Phys. Rev. Lett. 93 (2004) 090602, [arXiv:hep-th/0311175] .

[7] E. Shuryak, S.-J. Sin and I. Zahed, J. Korean Phys. Soc. 50 (2007) 384, [arXiv:hep-th/0511199].

[8] K.-Y. Kim, S.-J. Sin and I. Zahed, [arXiv:hep-th/0608046].

[9] N. Horigome and Y. Tanii, JHEP 0701 (2007) 072, [arXiv:hep-th/0608198]

[10] S. Nakamura, Y. Seo, S.-J. Sin and K. P. Yogendran, [arXiv:hep-th/0611021].

[11] S. Kobayashi, D. Mateos, S. Matsuura, R.C. Myers and R.M. Thomson, JHEP 0702 (2007) 016, [arXiv:hep-th/0611099].

[12] S. Nakamura, Y. Seo, S.-J. Sin and K. P. Yogendran, [arXiv:0708.2818[hep-th]].

[13] J. W. Chen, M. Huang, Y.H. Li, E. Nakano and D. L. Yang, Phys. Lett. B 670 (2008) 18, [arXiv:0709.3434 [hep-ph]]

[14] P. Kovtun, D. T. Son and A.O. Starinets, Phys. Rev. Lett. 94 (2005) 111601, [arXiv:hep-th/0405231] .

[15] Y. Kats and P. Petrov, JHEP 0901 (2009) 044 [arXiv:0712.0743[hep-th]].

[16] M. Brigante, H. Liu, R.C. Myers, S. Shenker and S. Yaida, Phys. Rev. D77 (2008) 126006, [arXiv:0712.0805[hep-th]].

[17] M. Brigante, H. Liu, R.C. Myers, S. Shenker and S. Yaida, Phys. Rev. Lett. 100 (2008) 191601, [arXiv:0802.3318[hep-th]].

[18] I.P. Neupane and N. Dahhich, Class. Quant. Grav. $26 \quad$ (2009) 015013. [arXiv:0808.1919[hep-th]]; I.P. Neupane, [arXiv:0904.4805[hep-th]].

[19] R. Brustein and A. M. Medved, [arXiv:0808.3498[hep-th]]

[20] R. Brustein and A. M. Medved, [arXiv:0810.2193[hep-th]] 
[21] N. Iqbal and H. Liu, [arXiv:0809.3808[hep-th]]

[22] N. Banerjee and S. Dutta, [arXiv:0903.3925[hep-th]]

[23] B, Zwiebach, Phys. Lett. B 156 (1986) 315

[24] B, Zumino, Phys. Rep. 137 (1986) 109

[25] D. Lovelock, J. Math. Phys. 12 (1971) 498

[26] X. H. Ge, Y. Matsuo, F.-W. Shu, S.-J. Sin and T. Tsukioka, JHEP 0810 (2008) 009, [arXiv:0808.2354[hep-th]]

[27] X. H. Ge and S.-J. Sin, JHEP 0905 (2009) 051 [arXiv:0903.2527[hep-th]]

[28] R. G. Cai and Y. W. Sun, JHEP 0603 (2008) 052, [arXiv:0807.2377[hep-th]]; R. G. Cai, Z. Y. Nie and Y. W. Sun, Phys. Rev. D78 (2008)126007 [arXiv:0811.1665[hep-th]]

[29] R. G. Cai, N. Ohta, Z. Y. Nie and Y. W. Sun, [arXiv:0901.1421[hep-th]]

[30] S. Cremonini, K. Hanaki, J. T. Liu and P. Szepietowski, [arXiv:0903.3244 [hep-th] ]

[31] R. C. Myers, M. F. Paulos and A. Sinha, [arXiv:0903.2834 [hep-th]]

[32] K. Bitaghsir Fadafan, JHEP 0812 (2008) 051, [arXiv:0803.2777 [hep-th]]; [arXiv: $0809.1336[$ hep-th] ]

[33] B. McInnes, [arXiv:0905.1180 [hep-th]]

[34] A. Buchel and R. Myers, [arXiv:0906.2922 [hep-th]]

[35] G. Dotti and R.J. Gleiser, Phys. Rev. D72 (2005) 044018, [arXiv:gr-qc/0503117]; R.J. Gleiser and G. Dotti, Phys. Rev. D72 (2005) 124002, [arXiv:gr-qc/0510069]; M. Beroiz, G. Dotti and R.J. Gleiser, Phys. Rev. D76 (2007) 024012, [arXiv:hep-th/0703074].

[36] R.A. Konoplya and A. Zhidenko, Phys. Rev. D77 (2008) 104004, [arXiv:0802.0267]. 
[37] T. Takahashi and J. Soda, Phys. Rev. D75 (2009) 104025, arXiv:0902.2921 [gr-qc]; arXiv:0907.0556 [gr-qc]

[38] M. H. Dehghani and M. Shamirzaie, Phys. Rev. D 72 (2005) 124015

[39] J. Crisostomo, R. Troncoso and J. Zanelli, Phys. Rev. D 62 (2000) 084013, [arXiv:hep-th/0003271]; R. Aros, R. Troncoso and J. Zanelli, Phys. Rev. D 63 (2001) 084015, [arXiv:hep-th/0011097].

[40] R. G. Cai, Phys. Lett. B 582 (2004) 237, [arXiv:hep-th/0311240]; R. G. Cai, N. Ohta, Phys.Rev. D74 (2006) 064001, [arXiv:hep-th/0604088]; R. G. Cai, L. M. Cao, Y. P. Hu and S. P. Kim, Phys.Rev.D 78 (2008)124012.

[41] M. H. Dehghani and N. Farhangkhah, Phys. Rev. D 78 (2008) 064015; M. H. Dehghani, N. Bostani and S. H. Hendi, Phys. Rev. D 78 (2008) 064031

[42] F.-W. Shu and X. H. Ge, JHEP 0808 (2008) 021 [arXiv:0804.2724 [hep-th]]; [arXiv:0804.2123 [hep-th]].

[43] D. Kastor and R. Mann, [arXiv:hep-th/0603168]

[44] X. H. Ge, Y. Matsuo, F.-W. Shu, S.-J. Sin and T. Tsukioka, Prog. Theor. Phys. 120 (2008) 833, arXiv:0806.4460[hep-th]].

[45] Y. Matsuo, S.-J. Sin, S. Takeuchi, T. Tsukioka and C. -M. Yoo, arXiv:0901.0610 [hep-th]].

[46] J. Troost, Phys. Lett. B 578 (2004) 210 , hep-th/0308044.

[47] B. Wang, C. Y. Li and E. Abdalla, Phys. Lett. B 481 (2000) 79 